\section{P2-355 FACTORS ASSOCIATED WITH OVERWEIGHT AND OBESITY IN INDIVIDUALS WITH HIV/AIDS: A MULTINOMIAL LOGISTIC REGRESSION ANALYSIS}

doi:10.1136/jech.2011.142976k.87

\begin{abstract}
${ }^{2} \mathrm{C}$ A Mariz, ${ }^{1} \mathrm{M}$ D F P Militão Albuquerque, ${ }^{* 2,3} \mathrm{R}$ Ximenes, ${ }^{2,3} \mathrm{H}$ R Lacerda, ${ }^{3} \mathrm{D}$ M Filho, ${ }^{4} \mathrm{~T}$ Gelenske, ${ }^{4} \mathrm{E}$ Carvalho, ${ }^{5} \mathrm{~A}$ P Silva, ${ }^{3} \mathrm{~F}$ Bandeira. ${ }^{1}$ Centro de pesquisas Aggeu magalhães, Fundação Oswaldo Cruz, Recife, Pernambuco, Brazil; ${ }^{2}$ Programa de PósGraduação em Medicina Tropical, Universidade Federal de Pernambuco, Recife, Pernambuco, Brazil; ${ }^{3}$ Universidade de Pernambuco, Recife, Pernambuco, Brazil; ${ }^{4}$ Instituto de Medicina Integral Prof. Fernando Figueira, Recife, Pernambuco, Brazil; ${ }^{5}$ Hospital Correia Picanço, Recife, Pernambuco, Brazil
\end{abstract}

Introduction The nutritional profile of HIV-infected patients after the introduction of HAART has pointed to high rates of overweight and obesity that have been associated with the emergence of long term co-morbidities, including metabolic changes.

Methods A cross-sectional nested case control study was conducted to estimate the prevalence of thinness and overweight/obesity and associated factors in 2018 individuals with HIV/AIDS, using the Body Mass Index (BMI). Thinness were classified as $\mathrm{BMI}<18.5 \mathrm{~kg} / \mathrm{m}^{2}$, overweight/obesity $\mathrm{BMI} \geq 25 \mathrm{~kg} / \mathrm{m}^{2}$ and eutrophic $B M I \geq 18.5-24.9 \mathrm{~kg} / \mathrm{m}^{2}$. As the outcome variable has more than two levels we performed the multinomial logistic regression analyses comparing thinness and overweight/obesity to eutrophic as the reference level.

Results The prevalence of thinness was $8.8 \%$ and that of overweight/obesity $32.1 \%$. The variables associated with thinness were anaemia and CD4 cell count $<200 \mathrm{~mm}^{3}$. The risk factors for overweight/obesity were age $\geq 40$ years and diabetes and the variables identified as protective factors for overweight/obesity were not having a long term partner, smoking, presence of an opportunistic disease, anaemia, and albumin levels $<3.5 \mathrm{mg} / \mathrm{dl}$.

Conclusion The main nutritional problem observed in this population was overweight and obesity, which were much more prevalent than thinness. Older individuals with diabetes should be targeted for nutritional interventions and lifestyle changes.

\section{P2-356 INDEPENDENT PREDICTORS OF METABOLIC SYNDROME IN HIV-INFECTED PATIENTS}

doi:10.1136/jech.2011.142976k.88

\begin{abstract}
1,2P R D Alencastro, *2,3S C Fuchs, 1,2M L R Ikeda, 3,4F H Wolff, ${ }^{4,5}$ A B M Brandão, ${ }^{3.4} \mathrm{~N}$ T Barcellos. ${ }^{1}$ Hospital Sanatório Partenon, Health Secretariat of State of Rio Grande do Sul, Porto Alegre, Rio Grande do Sul, Brazil; ${ }^{2}$ Postgraduate Studies Program in Cardiology, School of Medicine, Universidade Federal do Rio Grande do Sul, Porto Alegre, Rio Grande do Sul, Brazil; ${ }^{3}$ Postgraduate Studies Program in Epidemiology, School of Medicine, Universidade Federal do Rio Grande do Sul, Porto Alegre, Rio Grande do Sul, Brazil; ${ }^{4} 4$ National Institute for Science and Technology for Health Technology Assessment (IATS/CNPq), Hospital de Clinicas de Porto Alegre, Porto Alegre, Rio Grande do Sul, Brazil; ${ }^{5}$ Faculdade de Medicina, Universidade Federal de Ciências da Saúde de Porto Alegre, Porto Alegre, Rio Grande do Sul, Brazil
\end{abstract}

Introduction Metabolic Syndrome (MetS) is associated with development of type 2 diabetes mellitus and increased risk for cardiovascular disease. However, a few studies have assessed its prevalence and risk factors among HIV patients from developing countries.

Objective To identify independent risk factors for metabolic syndrome by the criteria of the American Heart Association/National Heart, Lung, and Blood Institute (AHA/NHLBI) among men and women HIV-infected.

Study design A cross-sectional study enrolled patients, aged 18 years or older, who sought to confirm the diagnosis or treatment in the outpatient service of a public healthcare center, in southern Brazil From June 2006 to December 2008, certified research assistants conducted interviews using standardised questionnaires and anthropometric measurements. Fasting blood sample was collected, use of highly active antiretroviral therapy (HAART) was ascertained, and Metabolic Syndrome was characterised by the criteria of AHA/NHLBI.

Results 1240, out of 1295 HIV-infected patients were included. MetS prevalence was $24.7 \%$, and similar among men and women. Among men, age, education, physical activity, body mass index (BMI) and HAART use were independently associated with MetS, while among women, there were associations with age, BMI, and use of protease inhibitors.

Conclusions High prevalence of metabolic syndrome was detected in men and women HIV-infected. In both genders, age and BMI were directly and independently associated with MetS. The association between the use of HAART and MetS was confirmed among men but not among women.

\section{P2-357 CIGARETTE SMOKING CESSATION DURING PREGNANCY AND SUSTAINABILITY 4 YEARS AFTER DELIVERY}

doi:10.1136/jech.2011.142976k.89

${ }^{1,2} \mathrm{E}$ Alves, ${ }^{*}{ }^{1,2} \mathrm{~S}$ Correia, ${ }^{1,2} \mathrm{H}$ Barros, ${ }^{1,2} \mathrm{~A}$ Azevedo. ${ }^{1}$ Department of Hygiene and Epidemiology, University of Porto Medical School, Porto, Portugal; ${ }^{2}$ Institute of Public Health - University of Porto (ISPUP), Porto, Portugal

Introduction Pregnancy is an opportunity to promote lifestyle changes and modify the impact of earlier social and economic determinants. Our main objective was to study how decisions about smoking cessation during pregnancy evolve 4 years after delivery.

Methods In 2005-2006, puerperae were recruited in public maternities of Porto to enter a birth cohort. Within the ongoing cohort's re-evaluation at 4 years, we studied 3519 women. Marital status, educational level, income, social class and employment status were socioeconomic surrogates. Smoking before and during pregnancy was self-reported after delivery. Prevalence ratios (PR) adjusted for appropriate confounders and 95\% CIs were computed using robust Poisson regression.

Results Overall, 995 (22.6\%) women were smokers. During pregnancy, $10.2 \%$ did not change their consumption, $3.8 \%$ reduced and sustained the reduction 4 years after delivery, $37.3 \%$ reduced but resumed, $17.3 \%$ stopped smoking and maintained, and $31.4 \%$ ceased smoking but resumed. The probability of smoking cessation decreased with age (30-34 years: $\mathrm{PR}=0.78 ; 95 \%$ CI 0.62 to 0.97 ) and gravidity (multigesta: $\mathrm{PR}=0.72 ; 95 \% \mathrm{CI} 0.56$ to 0.92 ), and increased with education ( $\geq 13$ years: $P R=1.44 ; 95 \%$ CI 1.16 to 1.79 ) and smoking initiation age ( $\geq 18$ years: $P R=1.32 ; 95 \%$ CI 1.07 to 1.61 ). The sustainability of smoking cessation 4 years after delivery was associated with age ( $\geq 35$ years: $P R=2.19 ; 95 \%$ CI 1.32 to 3.61), pregnancy complications ( $\mathrm{PR}=1.41 ; 95 \%$ CI 1.03 to 1.94$)$ and having a new pregnancy ( $\mathrm{PR}=1.14 ; 95 \% \mathrm{CI} 1.15$ to 2.33 )

Conclusions Higher socioeconomic status was associated with smoking cessation during pregnancy but not with its sustainability. Although almost all women reduced or stopped smoking during pregnancy, more than half returned to their usual consumption level after delivery. 